\title{
Popular Diets: Intermittent Fasting ${ }^{1}$
}

\author{
Michelle Yavelow, Daniela Rivero Mendoza, and Wendy Dahl'
}

Weight loss can be challenging. Starting a restrictive diet can be emotionally draining and difficult to adhere to (Harris et al. 2018). Is there a more effective way to lose weight? Does the timing and frequency of meals help to lose weight? This publication describes the benefits and risks of intermittent fasting for weight loss.

\section{What is intermittent fasting?}

Fasting is the complete avoidance of food or drink. Fasting has been practiced for religious observances throughout history. For example, during Ramadan, Muslims fast from sunrise to sunset every day for 30 days. Intermittent fasting includes a variety of eating patterns where fasting happens over specific time periods on a repeating basis (Anton et al. 2018). Recently, intermittent fasting has been considered as a means to promote weight loss (Cho et al. 2019, Harris et al. 2018, Welton et al. 2020). There is a variety of patterns of intermittent fasting. Some of the common patterns are described below in Table 1 .

Table 1. Intermittent fasting eating patterns.

\begin{tabular}{|l|l|}
\hline Fasting Pattern & Fasting Pattern Explanation \\
\hline Time-restricted feeding & Fast for 8 to 16 hours each day \\
\hline Alternate-day fasting & $\begin{array}{l}\text { Fast for } 24 \text { hours followed up by a full } \\
\text { day of unrestricted eating }\end{array}$ \\
\hline $\begin{array}{l}\text { Alternate-day modified } \\
\text { fasting }\end{array}$ & $\begin{array}{l}\text { Allows 25\% of calorie needs on the } \\
\text { fasting day }\end{array}$ \\
\hline Periodic fasting & $\begin{array}{l}\text { Fast for } 1 \text { to } 2 \text { days (may allow 25\% of } \\
\text { calorie needs) and unrestricted eating } \\
\text { the rest of the week }\end{array}$ \\
\hline Adapted from Anton et al. (2018) and Patterson et al. (2015)
\end{tabular}

\section{What are the potential health benefits of intermittent fasting?}

Weight loss is an important goal for some people who are overweight or obese. If you are overweight or obese, losing weight can decrease the chances of developing chronic diseases, such as cardiovascular disease and type 2 diabetes (Kritchevsky et al. 2015). Weight loss strategies often include continuous calorie restriction-a decrease in the number of calories a person eats each day. Research studies show that intermittent fasting is no more effective for weight loss than continuous calorie restriction (Cioffi et al. 2018, Harris et al. 2018, Roman et al. 2019). However, compared to consuming a usual diet with no restrictions, intermittent fasting results in modest weight loss of less than 10 pounds (Harris et al. 2018).

In individuals with type 2 diabetes, fasting during 2 days per week (500-600 kcal/day) improved weight, blood glucose measurements, and quality of life (Corley et al. 2018). However, for individuals with type 2 diabetes on glucose-lowering medications, fasting of any type increased the rate of hypoglycemia (low blood sugar) (Corley et al. 2018), which, if not treated, can lead to dizziness, weakness, seizures, loss of consciousness, and even death. Because there is very little evidence regarding the safety and effectiveness for weight loss in individuals with diabetes, intermittent fasting should only be considered under the advisement of a physician (Horne et al. 2020). Proper education and medication management by a health care professional is needed to reduce the risk of hypoglycemic

1. This document is FSHN20-47, one of a series of the Food Science and Human Nutrition Department, UF/IFAS Extension. Original publication date September 2020. Visit the EDIS website at https://edis.ifas.ufl.edu for the currently supported version of this publication.

2. Michelle Yavelow, former graduate student; Daniela Rivero Mendoza, Extension and research coordinator; and Wendy Dahl, associate professor, Food Science and Human Nutrition Department, UF/IFAS Extension; Gainesville, FL 32611.

The Institute of Food and Agricultural Sciences (IFAS) is an Equal Opportunity Institution authorized to provide research, educational information and other services

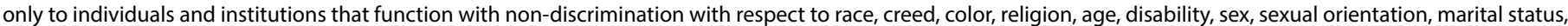

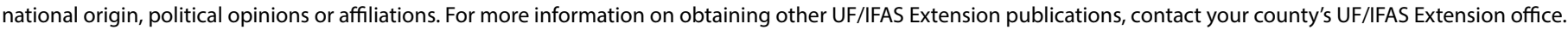
U.S. Department of Agriculture, UF/IFAS Extension Service, University of Florida, IFAS, Florida A \& M University Cooperative Extension Program, and Boards of County Commissioners Cooperating. Nick T. Place, dean for UF/IFAS Extension. 
events while practicing intermittent fasting (Corley et al. 2018).

In adults who are overweight or obese or those who do not have diabetes, intermittent fasting does not improve fasting blood glucose, insulin resistance, or LDL (bad) cholesterol (Cioffi et al. 2018, Harris et al. 2018). However, one review of studies reported a higher quality of life with intermittent fasting, compared to continuous energy restriction (Harris et al. 2018). Recent reviews of studies found that intermittent fasting and continuous calorie-restricted diets are more effective for the improvement of most cardiovascular health markers, such as blood cholesterol, compared to not dieting (Meng et al. 2020). In addition, a slight but significant reduction in fasting insulin has been reported for intermittent fasting, compared to continuous calorie restriction (Cioffi et al. 2018).

Intermittent fasting patterns with 6-hour or 12-hour feeding periods (meaning 12-hour to 18-hour fasting periods) were compared in men with prediabetes. The study was designed to provide the participants with enough food to maintain their body weight. The early time-restrictive feeding period improved insulin sensitivity and blood pressure (Sutton et al. 2018). Also, the early time-restrictive feeding period reduced appetite in the evening, despite the longer fasting duration (Sutton et al. 2018).

During weight loss, it is desirable to lose body fat while maintaining lean mass, that is, muscle mass. Reviews of research studies show that intermittent fasting does not preserve lean mass any more than continuous calorierestricted diets (Clifton 2017, Harris et al. 2018) and may even lead to a decrease in lean mass (Roman et al. 2019). Two reviews of research studies showed that loss of fat mass with intermittent fasting is similar to continuous calorierestricted diets (Cho et al. 2019), whereas one review of adults who were overweight and obese reported a beneficial effect for intermittent fasting (Harris et al. 2018). However, the level of adiponectin, a hormone associated with body fat breakdown and insulin sensitivity, may increase with intermittent fasting (Cho et al. 2019).

\section{What are the potential health risks of intermittent fasting?}

Few studies have been conducted to determine the long-term safety of intermittent fasting (Liu et al. 2020). Importantly, there are risks for certain populations, especially if individuals are not carefully followed by a health care professional. Most importantly, intermittent fasting may lead to hypoglycemia in individuals who have type
2 diabetes and are taking glucose-lowering medications (Corley et al. 2018).

Intermittent fasting may be hard for some people to adhere to (Harris et al. 2018), but with the variety of methods listed in the chart above, you may find a pattern that works with your lifestyle. Some health professionals have concerns that intermittent fasting may encourage disordered eating (Liu et al. 2020). Consequently, most research studies have excluded people with a medical history of eating disorders and psychiatric conditions (Liu et al. 2020). If you are currently or were previously diagnosed with these conditions, intermittent fasting may not be appropriate for you.

Metabolic Effects of Fasting
When you eat a meal, your body starts breaking down these
foods into smaller nutrients that can be used by your body. Your
body normally digests carbohydrates and uses glucose, a sugar,
as an energy source. The human body requires 130 grams of
glucose per day to keep the brain and nervous system functioning
well (Institute of Medicine and Food and Nutrition Board 2005).
However, fasting or consuming a very low carbohydrate intake
may prevent the body from obtaining sufficient glucose for energy
(Fedorovich, Voronina, and Waseem 2018). Instead, the body will
start burning fats as an energy source during fasting. This switch
will happen 12 to 32 hours after you begin fasting, depending on
how much carbohydrate is stored within your body. This switch will
happen faster if you exercise during a fasting period. Importantly,
some fasting rituals do not reach the point at which the body
starts to burn fats. For instance, in 2019 , Ramadan fast (an Islamic
tradition that includes fasting during the day) duration went from
11 hours to 20 hours depending on the latitude, so it is possible
that some observing the fast did not reach the switch (Al Jazeera
News 2010 ).

\section{Summary}

Studies have compared intermittent fasting to eating less every day and found that both result in similar weight loss. There is some evidence that intermittent fasting may improve insulin sensitivity and blood pressure independently of weight loss. However, there are risks related to intermittent fasting. Intermittent fasting poses a serious risk for hypoglycemia for individuals with type 2 diabetes who take glucose-lowering medications. For those who are currently or were previously diagnosed with eating disorders, intermittent fasting may not be recommended. Also, as with any other dietary pattern in which calorie intake may be limited, adherence to intermittent fasting can be challenging. The strength of evidence regarding the effects of intermittent fasting on weight and cardiometabolic risk factors is weak compared to the strong, consistent evidence for the health benefits of a Mediterranean dietary pattern (Dinu et al. 2020). 
Note: It is important to speak with your health care provider or registered dietitian nutritionist before making any changes to your diet.

\section{References}

Al Jazeera News. 2010. "Ramadan 2020: Fasting Hours around the World." Accessed June 15, 2020. https://www. aljazeera.com/indepth/interactive/2017/05/ramadan2017-fasting-hours-world-170511102015757.html.

Anton, Stephen D., Keelin Moehl, William T. Donahoo, Krisztina Marosi, Stephanie A. Lee, Arch G. Mainous III, Christiaan Leeuwenburgh, and Mark P. Mattson. 2018.

"Flipping the Metabolic Switch: Understanding and Applying the Health Benefits of Fasting." Obesity 26 (2): 254-268. https://doi.org/10.1002/oby.22065.

Cho, Yongin, Namki Hong, Kyung-won Kim, Sung joon Cho, Minyoung Lee, Yeon-hee Lee, Yong-ho Lee, Eun Seok Kang, Bong-Soo Cha, and Byung-Wan Lee. 2019. “The Effectiveness of Intermittent Fasting to Reduce Body Mass Index and Glucose Metabolism: A Systematic Review and Meta-analysis." Journal of Clinical Medicine 8 (10): 1645. https://doi.org/10.3390/jcm8101645.

Cioff, Iolanda, Andrea Evangelista, Valentina Ponzo, Giovannino Ciccone, Laura Soldati, Lidia Santarpia, Franco Contaldo, Fabrizio Pasanisi, Ezio Ghigo, and Simona Bo. 2018. "Intermittent versus Continuous Energy Restriction on Weight Loss and Cardiometabolic Outcomes: A Systematic Review and Meta-analysis of Randomized Controlled Trials." Journal of Translational Medicine 16 (1): 371. https:// doi.org/10.1186/s12967-018-1748-4.

Clifton, Peter. 2017. "Assessing the Evidence for Weight Loss Strategies in People with and without Type 2 Diabetes." World Journal of Diabetes 8 (10): 440-454. https://doi. org/10.4239/wjd.v8.i10.440.

Corley, B. T., R. W. Carroll, R. M. Hall, M. Weatherall, A. Parry-Strong, and J. D. Krebs. 2018. "Intermittent Fasting in Type 2 Diabetes Mellitus and the Risk of Hypoglycaemia: A Randomized Controlled Trial." Diabetic Medicine 35 (5): 588-594. https://doi.org/10.1111/dme.13595.
Dinu, Monica, Giuditta Pagliai, Donato Angelino, Alice Rosi, Margherita Dall'Asta, Letizia Bresciani, Cinzia Ferraris, Monica Guglielmetti, Justyna Godos, Cristian Del Bo', Daniele Nucci, Erika Meroni, Linda Landini, Daniela Martini, Francesco Sofi, and The Working Group "Young Members" of the Italian Society of Human Nutrition. 2020. "Effects of Popular Diets on Anthropometric and Cardiometabolic Parameters: An Umbrella Review of Meta-analyses of Randomized Controlled Trials." Advances in Nutrition 11 (4): 815-833. https://doi.org/10.1093/ advances/nmaa006.

Fedorovich, Sergei V., Polina P. Voronina, and Tatyana V. Waseem. 2018. "Ketogenic Diet versus Ketoacidosis: What Determines the Influence of Ketone Bodies on Neurons?" Neural Regeneration Research 13 (12): 2060-2063. https:// doi.org/10.4103/1673-5374.241442.

Harris, Leanne, Sharon Hamilton, Liane B. Azevedo, Joan Olajide, Caroline De Brún, Gillian Waller, Vicki Whittaker, Tracey Sharp, Mike Lean, Catherine Hankey, and Louisa Ells. 2018. "Intermittent Fasting Interventions for Treatment of Overweight and Obesity in Adults: A Systematic Review and Meta-analysis." JBI Evidence Synthesis 16 (2): 507-547. https://doi.org/10.11124/jbisrir-2016-003248.

Horne, B. D., M. M. Grajower, and J. L. Anderson. 2020. "Limited Evidence for the Health Effects and Safety of Intermittent Fasting among Patients with Type 2 Diabetes." JAMA 324 (4): 341-342. https://doi.org/10.1001/ jama.2020.3908.

Institute of Medicine, and Food and Nutrition Board. 2005. Dietary Reference Intakes for Energy, Carbohydrate, Fiber, Fat, Fatty Acids, Cholesterol, Protein, and Amino Acids. Washington, D.C.: The National Academies Press.

Kritchevsky, Stephen B., Kristen M. Beavers, Michael E. Miller, M. Kyla Shea, Denise K. Houston, Dalane W. Kitzman, and Barbara J. Nicklas. 2015. "Intentional Weight Loss and All-Cause Mortality: A Meta-analysis of Randomized Clinical Trials." PloS one 10 (3): e0121993-e0121993. https://doi.org/10.1371/journal.pone.0121993.

Liu, Kai, Bo Liu, and Leonie K Heilbronn. 2020. "Intermittent Fasting: What Questions Should We Be Asking?" Physiology \& Behavior 218:112827. 
Meng, Haiyan, Lei Zhu, Hamed Kord-Varkaneh, Heitor O. Santos, Grant M. Tinsley, and Peng Fu. 2020. "Effects of Intermittent Fasting and Energy-Restricted Diets on Lipid Profile: A Systematic Review and Meta-analysis." Nutrition 77:110801. https://doi.org/10.1016/j.nut.2020.110801.

Patterson, Ruth E., Gail A. Laughlin, Andrea Z. LaCroix, Sheri J. Hartman, Loki Natarajan, Carolyn M. Senger, María Elena Martínez, Adriana Villaseñor, Dorothy D. Sears, Catherine R. Marinac, and Linda C. Gallo. 2015. "Intermittent Fasting and Human Metabolic Health." Journal of the Academy of Nutrition and Dietetics 115 (8): 1203-1212. https://doi.org/10.1016/j.jand.2015.02.018.

Roman, Yuani M., Mariah C. Dominguez, Tommy M. Easow, Vinay Pasupuleti, C. Michael White, and Adrian V. Hernandez. 2019. "Effects of Intermittent versus Continuous Dieting on Weight and Body Composition in Obese and Overweight People: A Systematic Review and Meta-analysis of Randomized Controlled Trials." International Journal of Obesity 43 (10): 2017-2027. https:// doi.org/10.1038/s41366-018-0204-0.

Sutton, Elizabeth F., Robbie Beyl, Kate S. Early, William T. Cefalu, Eric Ravussin, and Courtney M. Peterson. 2018. "Early Time-Restricted Feeding Improves Insulin Sensitivity, Blood Pressure, and Oxidative Stress Even without Weight Loss in Men with Prediabetes." Cell Metabolism 27 (6): 1212-1221.e3. https://doi.org/10.1016/j. cmet.2018.04.010.

Welton, Stephanie, Robert Minty, Teresa O'Driscoll, Hannah Willms, Denise Poirier, Sharen Madden, and Len Kelly. 2020. "Intermittent Fasting and Weight Loss." Canadian Family Physician 66 (2): 117-125. 